\title{
Método Kaizen para optimizar la calidad del servicio postventa en una cadena de bienes durables, ciudad de Piura 2020.
}

\author{
Elmer Bagner Salazar Salazar ${ }^{1}$, Roberto Murillo Miranda $^{2}$
}

\begin{abstract}
The objective of this research was to design the Kaizen method to optimize the quality of the after-sales service in a chain of durable products in the city of Piura 2020. The methodology used was of an applied type, with a quantitative approach, it used a deductive method, it was a non-experimental and purposeful design. The sample consisted of 57 clients of a chain of durable products in the city of Piura, to whom a questionnaire with a Likert-type scale was applied which was validated through the judgment of experts and whose reliability yielded 0.784 in the Alpha coefficient of Cronbach. The results obtained were able to diagnose, decide and identify the most relevant factors for the descriptive analysis of both variables of the study carried out. Likewise, the proposal of the "Kokyaku" tool was supported as a new management tool to optimize the quality of the after-sales service. The most outstanding conclusions refer to the direct impact of Kaizen elements on the quality of after-sales service.
\end{abstract}

Keywords: Kaizen, quality of service, continuous improvement, durable products chain, after sales

\section{RESUMEN}

La investigación tuvo como objetivo diseñar el método Kaizen para optimizar la calidad del servicio postventa en una cadena de bienes durables, en la ciudad de Piura 2020. La metodología usada fue de tipo aplicada, con enfoque cuantitativo, usó método deductivo, fue un diseño no experimental y de nivel propositivo. La muestra fue de 57 clientes de una cadena de bienes durables en la ciudad de Piura, a quienes se les aplicó un cuestionario con escala de tipo Likert el cual fue validado mediante el juicio de expertos y cuya confiabilidad arrojó 0.784 en el coeficiente de Alfa de Cronbach. Los resultados obtenidos lograron diagnosticar, determinar e identificar los factores más relevantes para el análisis descriptivo de ambas variables del estudio realizado. Así mismo, fue sustentada la propuesta de la herramienta "Kokyaku" como una nueva herramienta de gestión para optimizar la calidad del servicio postventa. Las conclusiones más destacadas refieren el impacto directo de los elementos Kaizen sobre la calidad de servicio de postventa.

Palabras clave: Kaizen, calidad de servicio, mejora continua, cadena de bienes durables, postventa

Received: 10 de abril del 2021

Accepted: 05 de mayo de 2021

\section{Introducción}

En los últimos años las empresas asumieron el gran reto de ser rentables y competitivas en su entorno caótico, en donde los clientes idealizaron y exigieron mucho más por su dinero, debido a que, hubo diversidad de opciones que se ofrecieron para cubrir sus necesidades inmersas en un mundo globalizado. Esto obligó, por tanto, adopten un pensamiento holista que involucró el estudio de todas las variables asociadas a su macro y micro entorno. Además, los continuos avances tecnológicos, la evolución en los hábitos de los consumidores, la reducción de costos, la rapidez en los tiempos de respuesta y el escenario competitivo; explicaron la necesidad de aplicar métodos que faciliten enfrentar estos desafios.

Es por ello, que a nivel mundial la definición de calidad ha obtenido una mayor inclusión en cada organización. Muchos gerentes aceptaron la relevancia de cumplir con un servicio de calidad, para lograr un importante punto de quiebre en el negocio. Existieron muchas evidencias sobre el impacto directo de la calidad en el resultado de la gestión empresarial, la cual sobrepaso expectativas. La calidad en el servicio aumentó la fidelización en los clientes, estableció relaciones mucho más solventes, redujo la sensibilidad a los precios bajos, fomentó mayor motivación, etc. Los autores concluyeron que el concepto de calidad, fue la evaluación que el cliente realizó sobre la eficiencia en el diseño y la consistencia del servicio basado en las expectativas del cliente (Fernández y Bajac, 2018).

De igual forma, se buscó incentivar la gestión de mejora continua en el tipo de empresa comercializadora; mediante la realización de actividades que lograron articular el proceso de planificación, de implementación y de control de indicadores relacionados directamente con el éxito de la gestión. Asimismo, se orientó a la empresa hacia un sistema de filosof́a esbelta, que consistió en usar varias herramientas para reducir cualquier operación que no logre sumar valor al producto o servicio, incluyendo los procesos. Esto fue realizado tanto a nivel estratégico, como operativo; evitando desatender los requerimientos del entorno, la estrategia corporativa y las expectativas del cliente. Las organizaciones Implementaron un sistema de gestión asegurando los esfuerzos de transformación y de mejora continua. Por tanto, se enfocaron en actualizar la tecnologia para mejorar los procesos operativos y prepararon a su personal para

${ }^{1}$ Universidad de San Martín de Porres, Peru. E-mail:esalazars@usmp.pe 2 Universidad César Vallejo, Peru. E-mail:p7002445949@ucvvirtual.edu.pe

How to cite: Salazar, E., Murillo, R. (2021). Método Kaizen para optimizar la calidad del servicio postventa en una cadena de bienes durables, ciudad de Piura 2020. Revista de Análisis Económico y Financiero, Vol.4,N.2, 35-40. DOI: 10.15446/ing.investig.xxxx 
usarla, sin afectar el servicio que ofrecieron a los clientes (Carballo, Arellano y Ríos, 2018).

De igual forma, la incesante exigencia de los clientes por obtener mejor calidad a un menor precio y con una entrega a tiempo; dio como resultado que, quienes tan sólo priorizaron la exigencia de precios bajos, por reducción de costos, perdieron el diferencial de calidad y entrega puntual. El sistema de mejora continua que obtuvo mayor nivel de satisfacción a estos requerimientos corporativos, fue "Kaizen", cuya etimología provino de dos palabras japonesas que significaron: Imejora continua, gradual y ordenada" (Gestión, 2016).

Máynez, Cavazos y Valles (2016) en la investigación "Transferencia de conocimiento dentro de la empresa: Análisis de variables precursoras en un entorno leankaizen". Contribuyó con evidencia empírica acerca de las relaciones entre la cultura organizacional y la transferencia de conocimiento dentro de la empresa. Además, lo desarrolló en un contexto donde involucró el entendimiento de la necesidad del sistema Kaizen, el espíritu de trabajo y el compromiso afectivo organizacional. La investigación fue de tipo cuantitativa, empírica y de corte transversal. Como técnica se utilizó la encuesta, dirigido a 100 empleados, en una empresa ubicada en Ciudad de Juárez, Chihuahua. El estudio concluyó que, la cultura organizacional favoreció con el entendimiento de la utilidad del método kaizen, además, su impacto fue positivo en el espíritu y compromiso laboral.

Isea-Arguelles y Aldana-Zavala (2017) también mencionaron que la filosofía Kaizen, pudo ser aplicada en organizaciones donde se pretendió alcanzar el éxito por medio de la implementación de una filosof́a de gestión que fuera ideal con la visión de calidad total, esto implicó que el talento humano se formará gradualmente, para así, entender el proceso del aprendizaje continuo, lo cual permitió consolidar la eficiencia como visión de trabajo.

Zeithaml, Bitner y Gremler (2018) identificaron que las 5 dimensiones de calidad de servicio se desagregaron en 22 items: Elementos tangibles, se relacionó con equipos modernos, instalaciones atractivas, empleados con impecable apariencia y materiales con calidad visual; Fiabilidad, se involucró con cumplir lo prometido, demostrar sincero interés en ayudar al cliente, que la primera impresión fuera excelente, que el servicio se cumpla en el tiempo acordado y los registros estuvieran exentos de errores; Capacidad de respuesta, se vinculó con que se informe cuando concluyó el servicio, dar un servicio rápido, mucha disponibilidad para ayudar y tiempo disponible para resolver las inquietudes; Seguridad, se ligó con transmitir confianza a los clientes, dar seguridad en las transacciones con la empresa, ser siempre amables, tener los conocimientos suficientes para responder a las preguntas de los clientes; Empatía, se asoció con otorgar atención personalizada, tener horarios de trabajo convenientes, contar con personal que ofrece una atención individualizada, priorizar los mejores intereses de los clientes y comprender de las necesidades explicitas.

Se planteó un modelo basado en el sistema Kaizen y usarlo como instrumento de mejora, ante la deficiente calidad de servicio de las cadenas de bienes durables, en la ciudad de Piura. Se logró evidenciar que no hubo un profundo análisis en la definición del problema, con su respectivo diagnóstico actualizado y análisis de causas potenciales. Tampoco fue implementado un enfoque de mejora continua, con el seguimiento en la verificación de los resultados, y la estandarizaron de los procesos no se incluyeron en futuros planes. Finalmente, no se logró recabar valiosa información por parte del cliente. Como consecuencia encontramos que, hubo deficiente cuidado por la imagen de las instalaciones, equipos y personal. Así miso, el servicio no fue fiable, ni cuidadoso, e impactó en la credibilidad y confianza del servicio. Además, fue lenta la capacidad de respuesta hacia el cliente y finalmente, fue escaza la empatía entre la empresa y los clientes para establecer relaciones a largo plazo. En conclusión, no se obtuvo una nueva cultura de mejora continua, ni de trabajo en equipo.

La investigación se justificó, basado en que, se convirtió en una referencia importante para optimizar la calidad de servicio (variable dependiente), mediante herramientas Kaizen (variable independiente), en el segmento de bienes durables de la ciudad de Piura. Por tanto, se implementaron los elementos necesarios para fortalecer los procesos administrativos y orientarlos hacia el concepto de calidad total. Además, la presente investigación surgió ante la necesidad de optimizar y estandarizar el nivel de desempeño de servicio al cliente. (Rivera, 2013). Finalmente, porque fue necesario instruir una filosofía basada en la mejora continua, generando un nuevo habito conceptual y cultura organizacional. (Máynez, Judith Cavazos y Valles, 2016). Por lo antes mencionado, se formuló la siguiente interrogante: ¿Cómo diseñar el método Kaizen para optimizar la calidad del servicio postventa en una cadena de bienes durables en la ciudad de Piura 2020?.

Para poder responder a la interrogante se propuso como objetivo general: Diseñar el método Kaizen para optimizar la calidad del servicio postventa en una cadena de bienes durables en la ciudad de Piura 2020. Lo cual llevó a plantear los siguientes objetivos específicos: Diagnosticar la situación actual de las cadenas de bienes durables en la ciudad de Piura 2020; Determinar los factores del método Kaizen para optimizar la calidad del servicio postventa en una cadena de bienes durables en la ciudad de Piura 2020; Identificar los aspectos claves para evaluar la calidad del servicio postventa en una cadena de bienes durables en la ciudad de Piura 2020.

Así mismo, se planteó la siguiente hipótesis general: El método Kaizen permitirá optimizar la calidad del servicio postventa en una cadena de bienes durables en la ciudad de Piura 2020.

\section{Metodología / Modelo}

El presente estudio fue de tipo aplicada porque recogió información primaria para dar solución a problemas, mediante una propuesta y/o implementación. El enfoque de la investigación fue cuantitativo y fue apropiada porque estimó las magnitudes u ocurrencia de los fenómenos y comprobó las hipótesis, así mismo, realizó un proceso organizado de manera secuencial y probatorio. El método que se uso fue deductivo, el diseño fue no experimental y el nivel de la investigación fue Propositivo, porque elaboraron un marco teórico que diseñó una propuesta de gestión, que propuso ponerlo en práctica en el futuro y así permitió a 
la organización obtener un nivel de mejora continua en los procesos (Hernandez, Fernández y Baptista, 2014).

El muestreo para el presente estudio fue no probabilístico, debido a que no todos los elementos del universo tuvieron la misma probabilidad de ser parte de la muestra y estuvieron sujetos con las características o propósitos de la investigación. También, fue de tipo Accidental al ser seleccionado de manera fortuita (Navarro, 2014). Se calculo una muestra de 57 clientes de una cadena de bienes durables, pertenecientes a una población de 60 personas. Entre las técnicas y herramientas aplicadas están: Análisis FODA y matriz Ishikawa, usados para diagnosticar la situación actual de la cadena de bienes durables en la ciudad de Piura.

Análisis Documental con su ficha documentaria: para determinar cómo el método Kaizen logró optimizar la calidad del servicio postventa.

Entrevista y guía de entrevista: para identificar cuáles son los aspectos claves para medir el servicio postventa direccionada a Gerentes de cadena de bienes durables en la ciudad de Piura. Encuesta y cuestionario: Dirigido a la muestra de 57 clientes, que identificó los aspectos claves para evaluar la calidad de servicio postventa, y cuyas respuestas fueron diseñadas con la escala de Likert (Ñupas, Mejía, Novoa y Villagómez, 2014).

\section{Resultados}

Antes de proceder a mostrar los resultados vamos a realizar un análisis descriptivo de la variable dependiente calidad de servicio.

\section{Calidad de servicio}

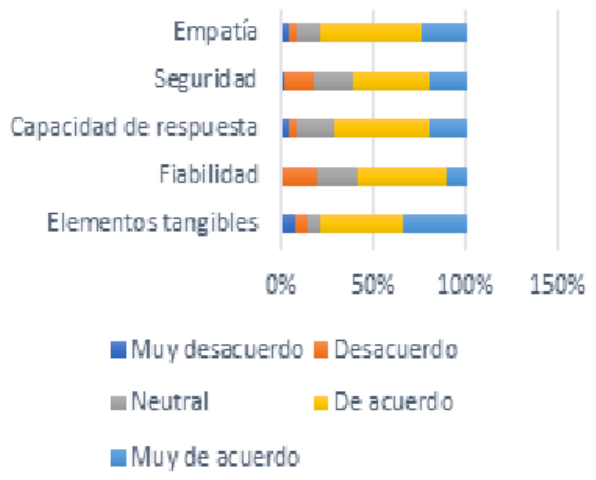

Figura 1. Gráfica de calidad de servicio

Se pudo observar el 79\% aprobó los elementos tangibles, el $59.6 \%$ la fiabilidad, el $71.9 \%$ la capacidad de respuesta, el $61.4 \%$ la seguridad y finalmente, el $79 \%$ la empatía.

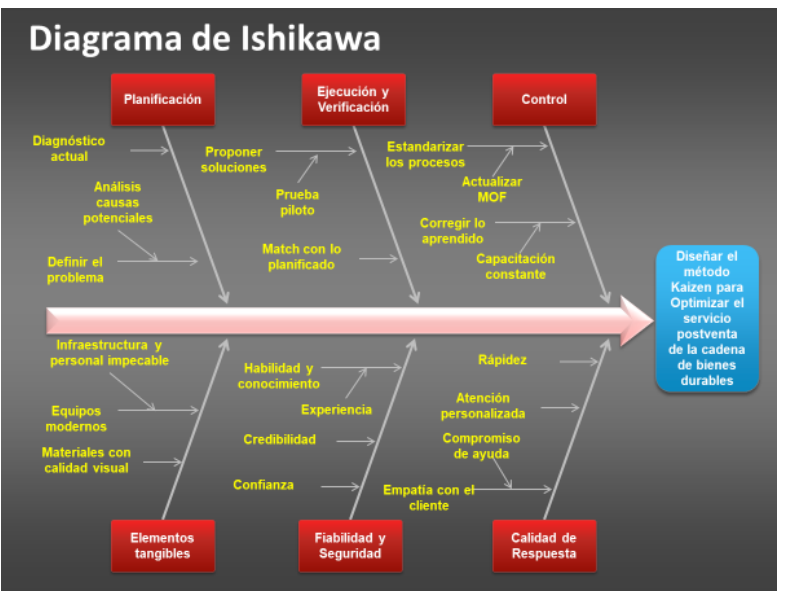

Figura 2. Matriz causa y efecto de Ishikawa

El presente diagrama Ishikawa contempló las siguientes 6M: Método (se relacionó con Control), Medición (se asoció a ejecución y verificación), Maquinaria (se ligó con Elementos tangibles), Management (se involucró con planificación), Gente (se enlazó con calidad de respuesta) y finalmente, Mantenimiento (se conectó a fiabilidad y seguridad). El diagrama reflejó como resultado diseñar el método Kaizen para poder optimizar el servicio postventa de la cadena de bienes durables como parte del diagnóstico realizado en la ciudad de Piura.

Se logró determinar los factores del método Kaizen para optimizar la calidad del servicio postventa en una cadena de bienes durables: Delimitar la magnitud del problema, establecer metas, objetivos y prioridades. Enfoque adecuado de los recursos para cumplir los objetivos. Acoplar las estrategias del FODA cruzado. Orientar la acción de los miembros de la organización. Buscar las oportunidades de cambio y mejora. Ejecutar lo planificado. Realizar pruebas piloto. Optimizar el uso de los recursos y habilidades. Fortalecer las herramientas de retroalimentación (InternaExterna). Comprobar la ejecución y registrarlo. Evaluar la funcionalidad de los recursos. Auditorías internas constantes. Actualizar las herramientas de gestión y el MOF. Seleccionar lo que generó valor y desechar lo contrario. Corregir y mejorar el resultado de los procesos. Introducir lo aprendido en nuevos programas de capacitación.

Las entrevistas identificaron los siguientes aspectos claves para evaluar la calidad de servicio postventa en una cadena de bienes durables: Los elementos tangibles, la buena visualización y equipos modernos, porque impactaron positivamente en la imagen de la organización; La fiabilidad fue sustentada en la rapidez, los procedimientos y la mejor actitud para fortalecer la fidelización de los clientes; Motivó el compromiso del empleado con capacitación constante e incentivos tangibles; También la seguridad que transmitió el empleado a sus clientes (postura, lenguaje, conocimiento, técnica); Y se enfocó en la importancia de las necesidades del cliente para lograr establecer relaciones a largo plazo. 


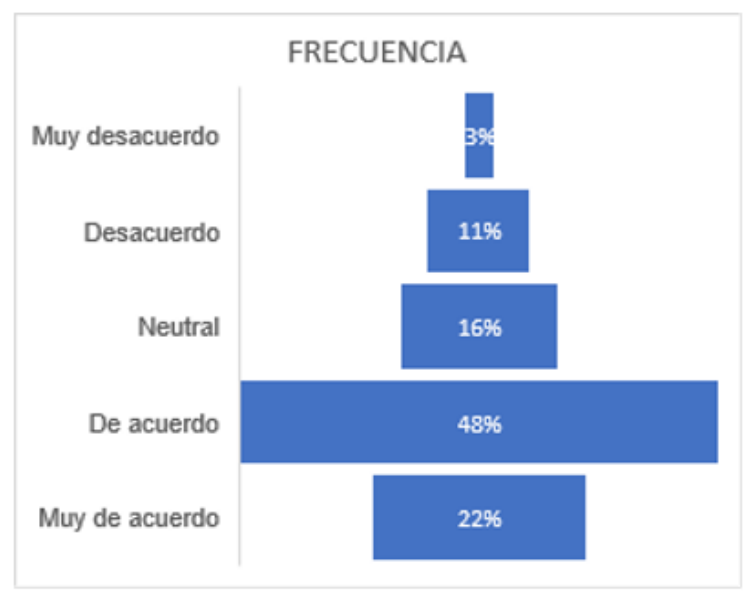

Fuente: cuestionario. Elaboración propia

Figura 3. Gráfica de la frecuencia porcentual

En términos generales los 57 clientes encuestados, reflejaron en un $70 \%$ que estuvieron conformes con los elementos que ayudaron a evaluar la calidad de servicio postventa en una cadena de bienes durables: Instalaciones, la fiabilidad y el cuidado del servicio, la actitud y voluntad del empleado, las habilidades del personal, la trascendencia de la confianza y credibilidad, finalmente, la atención personalizada. Así de esta manera, reforzó lo obtenido en el cuestionario. El 79\% aceptó que los equipos tangibles, tales como instalaciones, equipos, el personal y los materiales, impactaron directamente en forma positiva sobre la calidad de servicio. No obstante, el $14 \%$ no lo consideró así. Y el $7 \%$ se mantuvo neutral.

El 19.3\% no consideró que el servicio es fiable y cuidadoso. Mientras que, el $59.6 \%$ si consideró que el servicio prometido debe ser fiable y cuidadoso. Sólo $21.1 \%$ se mantuvo neutral. El $71.9 \%$ aprobó que la actitud y voluntad de los empleados es muy importante. Sólo un 8.8\% no aprobó este concepto y un $19.3 \%$ se mantuvo neutral.

El $61.4 \%$ percibió que las habilidades de los empleados ofrecen confianza y credibilidad en el servicio. Sin embargo, el $17.5 \%$ no lo percibió y el $21.1 \%$ se mantuvo neutral

El 79\% aceptó satisfactoriamente la atención personalizada como medio para generar una buena relación con el cliente. Sin embargo, el $8.8 \%$ no lo aceptó. Y el $12.3 \%$ se mantuvo neutral

Tabla 1. Confiabilidad del instrumento de recolección de datos

Coeficiente de Alfa de Cronbach en prueba piloto

\begin{tabular}{cc}
\multicolumn{2}{c}{ Estadísticas de fiabilidad } \\
Alfa de Cronbach & $\mathrm{N}$ de elementos \\
0.856 & 10
\end{tabular}

Coeficiente de Alfa de Cronbach en total encuestados Estadísticas de fiabilidad Alfa de Cronbach $\mathrm{N}$ de elementos 0.784 57

Fuente: Tomada de SPSS 22

Se Propone una nueva herramienta la cual se llamará "Kokyaku" cuyo significado se enfoca en la palabra Clientes, y está basada en los fundamentos Kaizen y enfocada en mejorar la calidad de servicio postventa en cadenas de bienes durables:

a) Soportada en 3 bases fundamentales: Base 1, estandarización y mejora continua de los procesos; Base 2, eliminación de todo lo que no genera valor; y Base 3, disciplina laboral mediante el aprendizaje.

b) Estructurada en 7 etapas bien definidas: 1) Identificar necesidades; 2) Definir metas, criterios y procesos; 3 ) Eliminar restricciones; 4) Generar, verificar y evaluar alternativas; 5) Seleccionar la mejor alternativa; 6) Estandarizar la mejora; 7) Seguimiento y capacitación continua.

c) Contempla las dimensiones de ambas variables del estudio, Kaizen (PHVA) y Calidad de servicio (Elementos tangibles, Fiabilidad, Capacidad de respuesta, Seguridad y Empatía).

d) Estrategia de 7s, ya no de 5s; Seiri (Clasificar), Seiton (Organizar), Seiso (limpiar obstáculos), Soryashon (Soluciones), Sabisu (Servicio), Seiketsu (Estandarizar, mantener) y finalmente, Shitsuke (Disciplina).

La presente matriz se fundamentó en el método Kaizen y articulo los conceptos teóricos y los fundamentos de esta filosofia para proponer una nueva herramienta de gestión orientada a la satisfacción del cliente en el servicio de postventa.

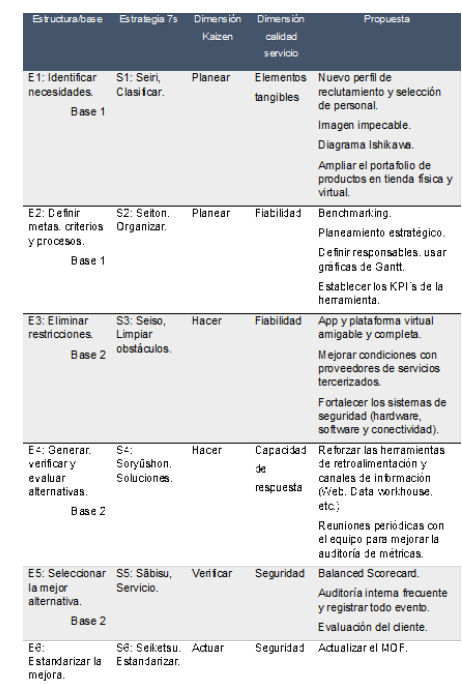

Figura 4. Matriz "Kokyaku" de calidad de servicio postventa

La actual propuesta fue diseñada para contribuir con la teoría existente sobre como implementar una herramienta Kaizen, en esta oportunidad se amplió el concepto a una estrategia de $7 \mathrm{~s}$, donde se involucraron las soluciones propuestas y el servicio. La propuesta, además, fue focalizada como un verdadero apoyo de gestión específico para el servicio postventa de cualquier cadena de bienes durables en la ciudad de Piura. 


\section{Discusión}

Con referencia al primer objetivo específico que consistió en diagnosticar la situación actual de las cadenas de bienes durables en la ciudad de Piura 2020, a través de un análisis FODA y diagrama de Ishikawa se evidenció la ausencia de estructura tecnológica, procesos, estandarización, compromiso del personal y estrategias para optimizar el servicio de postventa; como confirmó Carballo et al., (2018) sobre la importancia del uso de tecnología para mejorar los procesos operativos y preparar a su personal para usarla, sin que se afectará el servicio que se ofreció a los clientes. De igual forma resaltó Oropesa y García (2014) acerca de cómo el método Kaizen facilitó obtener una mejor calidad y productividad; y proporcionó evidencias claras en alcanzar mayor compromiso de los empleados, lo cual fue muy relevante para desarrollar una filosofía de cambio en el servicio postventa de las cadenas de bienes durables. Así mismo, Máynez et al. (2016) expresó la prioridad de generar una filosofía basada en la mejora continua, generando un nuevo habito conceptual y cultura organizacional. $Y$ lo comentado por Rivera (2013) sobre la necesidad de optimizar y estandarizar el nivel de desempeño de servicio al cliente.

En la discusión del segundo objetivo específico que determinó los factores del método Kaizen para optimizar la calidad del servicio postventa en una cadena de bienes durables de la ciudad de Piura 2020, fue realizado para ello un análisis descriptivo, mediante, una ficha documentaria; donde se evidenció estrategias y acciones que derivaron de las dimensiones e indicadores de la variable Kaizen (PHVA), para maximizar el desempeño en la calidad del servicio postventa. Donde surgieron factores como: análisis de causas potenciales, búsqueda de oportunidades de cambio, herramientas de retroalimentación, paneles de control y medición, articular sistemas de evaluación de desempeño, verificar resultados, establecer indicadores adecuados, estandarizar los procesos y programas de capacitación continua; tan igual como lo señaló Guerrero (2019) al evidenciar que el método Kaizen mejoró la calidad de los servicios; porque optimizó todos los procesos, con menores tiempos operativos y alcanzó los objetivos institucionales de forma oportuna. De igual manera, Olivares (2016) definió a Kaizen como la estrategia de Imejora continua" en la empresa y se focalizó en los factores $\mathrm{P}-\mathrm{H}-\mathrm{V}-\mathrm{A}$, involucrando a todo el personal y estandarizando los procesos. Por su lado Toscano, Brito, Magaña y González (2019) definieron la vital presencia y utilidad de Kaizen en cada fase, desde la recepción de la orden, pasando por la planificación, revisión, hasta llegar al embarque y lograr una estructura de proceso esbelta. Además, Cogollo, Zapa, Díez y Loayza (2018) graficó los factores relevantes que impactan en la cultura organizacional y obtuvo un mejor resultado transversal en productividad y competitividad de la empresa.

Respecto, al tercer objetivo específico se identificó los aspectos claves para evaluar la calidad del servicio postventa en una cadena de bienes durables en la ciudad de Piura 2020, se evidencio con el uso de entrevistas a Gerentes de cadenas de servicio y un cuestionario al grupo muestra de 57 clientes de cadenas de bienes durables, que los aspectos claves coinciden con las dimensiones descritas en el presente estudio relacionadas a la calidad de servicio y son: Elementos tangibles, obtuvo un 79\% de elección; Fiabilidad del servicio, reflejo un 59.6\% de aceptación; Capacidad de respuesta, registró un $71.9 \%$ de aprobación; Seguridad que siente el cliente de parte de la empresa, mostró un $61.4 \%$ de concordancia; y la empatía como elemento de relaciones a largo plazo, indicó un 79\% en su aceptación. Los resultados en general en su frecuencia dieron un $70 \%$ de aprobación en los aspectos claves mencionados anteriormente y con una confiabilidad de $\mathbf{0 . 7 8 4}$ en el Alfa de Cronbach para el cuestionario. En mención a lo anterior, se pudo relacionar con lo expresado por Fernández y Bajac (2018) al asociar la calidad en el servicio con la fidelización en los clientes, relaciones mucho más solventes, menor sensibilidad a los precios bajos y mayor motivación tanto del cliente como del personal. Así también, según Mendoza (2009) identificó claramente como aspectos claves y dimensiones de la calidad de servicio a: Elementos Tangibles, como la apariencia de las instalaciones, equipos, empleados y materiales de comunicación; Fiabilidad, como la habilidad para dar el servicio prometido de forma correcta; Capacidad de respuesta, como el desprendimiento que hubo para ayudar a los cliente de forma rápida; Seguridad, como el conocimiento y habilidades adquiridas por los empleados para transmitir confianza; y empatía, como la atención personalizada ofrecida. Igualmente, Zapata-Gómez (2014) lo graficó en su tabla de dimensiones de calidad de servicio y Zeithaml, Bitner y Gremler (2018) identificaron las mismas 5 dimensiones, que incluso se desagregaron en 22 items.

Finalmente, con el propósito de aportar con el objetivo general de diseñar el método Kaizen para optimizar la calidad del servicio postventa en una cadena de bienes durables en la ciudad de Piura 2020, se elaboró una herramienta de gestión basada y fundamentada en los principios Kaizen, que facilitó implementar procesos más dinámicos, adicionó valor en la gestión, aprovechó toda oportunidad de cambio con mejora, previno futuros errores y estableció relaciones duraderas con los clientes. Adicionalmente, la propuesta diseñada fue llamada "Kokyaku" la cual consideró establecer 7 etapas del proceso de cambio, donde se involucró y articuló muy bien las 3 bases teóricas fundamentales de Kaizen, las dimensiones de ambas variables en estudio, y se elaboró un tablero de $7 \mathrm{~S}$ como nueva técnica para el presente modelo de calidad en servicio postventa de una cadena de productos durables, esto lo se pudo sustentar con lo descrito por los autores Silke y Landisoa (2015) que señalaron la prioridad de responder con una estrategia adecuada que permita desarrollar mecanismos de recuperación del servicio para lograr clientes satisfechos. Por otro lado, tuvimos a Mañay (2016) quien implementó varias herramientas para mejorar cada uno de los procesos y fueron estandarizados con retroalimentación permanente. Por consiguiente, fue conocido también, como mejora continua. Además, Zelada (2017) destacó que la técnica de las 5'S logró superiores rangos de seguridad, excelente motivación de los empleados, óptima calidad, tiempos de respuesta rápidos, amplia vida útil de los elementos tangibles, nueva cultura organizacional y mejor control de costos.

\section{Conclusiones}

Primera: Al diagnosticar la situación actual de las cadenas de bienes durables en la ciudad de Piura, se concluyó que se debió considerar e involucrar los conceptos del 
método Kaizen, como tomar avances en materia tecnológica, desarrollar estrategias novedosas ante la evolución de los hábitos de los consumidores, mejorar los tiempos de respuesta y mayor dinamismo en los procesos; para obtener una mejor calidad de servicio postventa en dichas cadenas.

Segunda: Para determinar los factores del método Kaizen para optimizar la calidad del servicio postventa en una cadena de bienes durables en la ciudad de Piura, se concluyó que es relevante desarrollar alternativas de cambio y asociarlos a sus respectivas estrategias y acciones para rendir el máximo provecho de las dimensiones e indicadores del método Kaizen. Así mismo, fortalecer cada etapa del procedimiento Planear, Hacer, Verificar y Actuar.

Tercera: Al identificar los aspectos claves para evaluar la calidad del servicio postventa en una cadena de bienes durables en la ciudad de Piura, se concluyó que los aspectos claves fueron claramente definidos como los elementos tangibles, la fiabilidad del servicio, la capacidad de respuesta, la seguridad y la empatía del servicio. Con una confiabilidad de 0.784 en el Alfa de Cronbach.

Cuarta: En cuanto al objetivo general de diseñar el método Kaizen para optimizar la calidad del servicio postventa en una cadena de bienes durables en la ciudad de Piura, se concluyó que la propuesta "Kokyaku" contiene todos los elementos para realizar una excelente gestión de calidad en el servicio postventa de cualquier cadena de bienes durables. Dando así mismo, por demostrada la hipótesis general.

\section{Agradecimientos}

Los autores agradecen al apoyo del Instituto de Investigación de la Facultad de Ciencias Contables y Económicas y Financiceras de la Universidad San Martín de Porres.

\section{References}

Carballo, B., Arellano, A., Ríos, N. (2018). La gestión de procesos esbeltos como principio de mejora. Un caso aplicado a una comercializadora. 3C Empresa: Investigación y pensamiento crítico.

Cogollo, J. Zapa, E. Díez, V. Loayza, Loayza, O. (2018). Relación entre Kaizen y cultura laboral en sistemas productivos. Revista Espacios. Vol 39, Año 2018, Número 14, Pág. 10.

Fernández, P. Bajac, H. (2018). La gestión del marketing de servicios: principios y aplicaciones para la actividad gerencial. México, Chile, Argentina, España, Uruguay: Ediciones Granica S.A.

Gestión. (2016). Modelo Kaizen. Obtenido de http://gestioncalidad.com/modelo-kaizen-costes-calidad

Guerrero, J. (2019). El método kaizen para orientar la gestión logística en el cuartel general del Ejército Peruano- Sullana, año 2018 [tesis para Administración, Universidad Privada César Vallejo]. Repositorio Institucional UCV.

Hernández, R., Fernández, C., Baptista, M.(2014).Metodología de la investigación (6ta ed.). México: McGraw-Hill.

Isea-Arguelles, J. Aldana-Zavala, J. (2017). Filosofía gerencial Kaizen dirigido al Talento humano con responsabilidades gerenciales administrativas. Revista Cientifica FIPCAEC (Fomento de la investigación y publicación en Ciencias Administrativas, Económicas y Contables). Polo de
Capacitación, Investigación y Publicación (POCAIP): Vol. 2 , núm. 3.

Mañay, J. (2016). Propuesta de instructivo Kaizen para el mejoramiento continuo en las Pymes manufactureras del D.M.Q. caso: CIIU (31) [tesis para Ingeniería Comercial, Pontificia Universidad Católica del Ecuador]. Repositorio Institucional PUCE.

Máynez, A., Judith Cavazos, J., Valles, L. (2016). Transferencia de conocimiento dentro de la empresa: análisis de variables precursoras en un entorno lean-kaizen.

Mendoza J. (2009). Medición de la calidad del servicio. El Cid Editor.

Navarro, J. (2014). Epistemología y Metodología de la Investigación. México: Editorial patria.

Ñaupas, H., Mejía, E., Novoa, E., Villagómez, A. (2014). Metodología de la investigación cuantitativa - cualitativa y redacción de la tesis (4ta ed.). Colombia: Ediciones de la U.

Olivares, O. (2016).Aplicación como estrategia del KAIZEN en la empresa ópera form.

Oropesa, M., García, J. (2014). Beneficios del Kaizen en la Industria. I Congreso de Gestión de la Calidad y Protección Ambiental, Habana. Cuba. n_en_la_Industria.

Rivera, L. (2013). Justificación conceptual de un modelo de implementación de Lean Manufacturing. Heurística, No. 15-2013, 2013 - academia.edu.

Silke, R. y Landisoa, E. (2015). Service recovery for moderate and high involvement services. Journal of Services Marketing, Vol. 29, núm. 5, pp. 331-343. DOI: 10.1108 / JSM-05-2014-0155

Toscano, I., Brito, E., Magaña, S., González, M. (2019). Homeostasis of the manufacturing industry in Jalisco, México: kaizen as a negentropy in shipping logistics.

Zapata-Gómez, A. (2014). Application of structural equation and servqual in a health service. Dyna, Vol. 81, núm. 186, pp. 166-174.

Zeithaml, V., Bitner, M., Gremler, D. (2018). Marketing de servicios: integración del enfoque en el cliente en toda la empresa - 7 ed. Educación: McGraw- Hill.

Zelada, C. (2017). Propuesta de mejora de los procesos de servicio postventa de una empresa comercializadora de bienes de capital [tesis de maestría, Universidad Peruana de Ciencias Aplicadas]. Repositorio Institucional UPC. 